\title{
EIGENELEMENTS OF PERTURBED OPERATORS
}

\author{
B. V. LIMAYE and M. T. NAIR
}

(Received 23 January 1989)

Communicated by S. Yamamuro

\begin{abstract}
Let $\lambda_{0}$ be a semisimple eigenvalue of an operator $T_{0}$. Let $\Gamma_{0}$ be a circle with centre $\lambda_{0}$ containing no other spectral value of $T_{0}$. Some lower bounds are obtained for the convergence radius of the power series for the spectral projection $P(t)$ (and for trace $T(t) P(t)$ ) associated with a linear perturbation family $T(t)=T_{0}+t V_{0}$ and the circle $\Gamma_{0}$. They are useful when $T_{0}$ is a member of a sequence $\left(T_{n}\right)$ which approximates an operator $T$ in a collectively compact manner. These bounds result from a modification of Kato's method of majorizing series, based on an idea of Redont. If $\lambda_{0}$ is simple, it is shown that the same lower bounds are valid for the convergence radius of a power series yielding an eigenvector of $T(t)$.
\end{abstract}

1980 Mathematics subject classification (Amer. Math. Soc.) (1985 Revision): 47 A 55, 47 A 70, 47 A 10, 41 A 35 .

\section{Introduction}

Let $T_{0}$ and $V_{0}$ be closed (linear) operators on a domain $D_{0}$, which is a dense subspace of a Banach space $X$ over the field $\mathbb{C}$ of complex numbers. For $t \in \mathbb{C}$, consider the operator

$$
T(t)=T_{0}+t V_{0}
$$

Then $T(0)=T_{0}$ is called the unperturbed operator, $t V_{0}$ the perturbation and $T(t)$ the perturbed operator. This situation typically arises in quantum mechanics as follows. Let $T_{0}$ be the Hamiltonian of a quantum mechanical system and $V_{0}$ be a potential energy operator. Then $T(1)=T_{0}+V_{0}$ is the Hamiltonian of the perturbed system. Also, in operator approximation

(C) 1990 Australian Mathematical Society 0263-6115/90 \$A2.00+0.00 
theory, one considers a sequence of operators $\left(T_{n}\right)$ which approximates a given operator $T$. One can let $T_{0}=T_{n}$ for some fixed integer $n$ and $V_{0}=T-T_{0}$, so as to have $T=T(1)$.

Let $\sigma\left(T_{0}\right)$ denote the spectrum of $T_{0}$ and $\lambda_{0}$ be an isolated point of it. Let $\Gamma_{0}$ be a simple closed curve which separates $\lambda_{0}$ from the rest of $\sigma\left(T_{0}\right)$. For $z \notin \sigma\left(T_{0}\right)$, let $R_{0}(z)=\left(T_{0}-z I\right)^{-1}$. Consider the following open disk in $\mathbb{C}$ :

$$
\partial\left(\Gamma_{0}\right)=\left\{t \in \mathbb{C}:|t|<\min _{z \in \Gamma_{0}}\left(1 / r_{\sigma}\left(V_{0} R_{0}(z)\right)\right\},\right.
$$

where $r_{\sigma}$ denotes the spectral radius. For all $t \in \partial\left(\Gamma_{0}\right)$, it follows that $T(t)$ is a closed operator on the domain $D_{0}$, and $\Gamma_{0}$ does not intersect the spectrum $\sigma(T(t))$ of $T(t)$. For $z \notin \sigma(T(t))$, let $R(t, z)=(T(t)-z I)^{-1}$. Then the spectral projection

$$
P(t)=-\frac{1}{2 \pi i} \int_{\Gamma_{0}} R(t, z) d z
$$

associated with $T(t)$ and $\Gamma_{0}$, is an analytic function on $\partial\left(\Gamma_{0}\right)$. If the algebraic multiplicity of $\lambda_{0}$ is $m<\infty$, that is, if $P(0)$ has rank $m$, then for all $t \in \partial\left(\Gamma_{0}\right), P(t)$ has rank $m$ and $\Gamma_{0}$ contains precisely $m$ eigenvalues of $T(t): \lambda_{1}(t), \ldots, \lambda_{m}(t)$, repeated according to their algebraic multiplicities. Also, the function

$$
\hat{\lambda}(t) \equiv \frac{1}{m} \operatorname{trace} T(t) P(t)=\left(\lambda_{1}(t)+\cdots+\lambda_{m}(t)\right) / m
$$

is analytic for $t$ in $\partial\left(\Gamma_{0}\right)$.

It is of interest to know for which values of the parameter $t$, the power series for $P(t)$ and $\hat{\lambda}(t)$ converge. The method of majorizing series developed by Kato [2, pages 89-91] allows one to find lower bounds for the convergence radii of these series. A modification of Kato's method yields improved results for the case where $\lambda_{0}$ is a semisimple eigenvalue of $T_{0}$ (Lemma 2.1 and Remark 2.2). This procedure also gives an upper bound for $\left|\lambda_{i}(t)-\lambda_{0}\right|, i=$ $1, \ldots, m$, and a disk of isolation for the set $\left\{\lambda_{1}(t), \ldots, \lambda_{m}(t)\right\}$ from the rest of the spectrum of $T(t)$ (Theorem 2.3). The basic idea behind these results is given in Redont's unpublished work [7, pages 79-80] (compare also [6, Proposition 2.1] and [4, Theorem 10.5]. The results are applicable when a compact operator $T$ is approximated in a collectively compact manner (Remark 2.5).

In case $\lambda_{0}$ is a simple eigenvalue of $T_{0}$, let $\phi_{0}$ (respectively $\phi_{0}^{*}$ ) be an eigenvector of $T_{0}$ (respectively $T_{0}^{*}$ ) corresponding to $\lambda_{0}$ (respectively $\overline{\lambda_{0}}$ ) such that $\left\langle\phi_{0}, \phi_{0}^{*}\right\rangle=1$, and consider the meromorphic function

$$
\phi(t)=P(t) \phi_{0} /\left\langle P(t) \phi_{0}, \phi_{0}^{*}\right\rangle \text {. }
$$


If $t_{0} \in \partial\left(\Gamma_{0}\right)$ is not a pole of the function $\phi$, then $\phi\left(t_{0}\right)$ is an eigenvector of $T\left(t_{0}\right)$.

We describe a subregion of $\partial\left(\Gamma_{0}\right)$, which is devoid of any pole of the function $\phi$ (Proposition 3.2). This again yields a lower bound for the convergence radius of the power series for $\phi(t)$ about 0 (Theorem 3.3 and Remark 3.4).

\section{Eigenvalues of $T(t)$ near $\lambda_{0}$}

We assume that $\lambda_{0}$ is a semisimple eigenvalue of $T_{0}$ of algebraic multiplicity $m$, that is, the range of the spectral projection $P(0)=P_{0}$ coincides with the eigenspace of $T_{0}$ corresponding to $\lambda_{0}$ and is of dimension $m$. Let $S_{0}$ denote the reduced resolvent associated with $T_{0}$ and $\lambda_{0}$ :

$$
S_{0}=\lim _{z \rightarrow \lambda_{0}} R_{0}(z)\left(I-P_{0}\right)
$$

Then $S_{0 \mid\left(I-P_{0}\right) D_{0}}$ is the inverse of $\left(T_{0}-\lambda_{0} I\right)_{\mid\left(I-P_{0}\right) D_{0}}$. Since $\sigma\left(T_{0 \mid\left(I-P_{0}\right) D_{0}}\right)=$ $\sigma\left(T_{0}\right) \backslash\left\{\lambda_{0}\right\}$, it follows that

$$
\frac{1}{\operatorname{dist}\left(\lambda_{0}, \sigma\left(T_{0}\right) \backslash\left\{\lambda_{0}\right\}\right)}=r_{\sigma}\left(S_{0}\right) \leq\left\|S_{0}\right\| .
$$

Let $0<\varepsilon<1$. Consider the circle $\Gamma_{\varepsilon}$ with centre $\lambda_{0}$ and radius $\varepsilon /\left\|S_{0}\right\|$. The convergence radii of the series for $P(t)$ and $\hat{\lambda}(t)$ are greater than or equal to the radius of $\partial\left(\Gamma_{\varepsilon}\right)$. To find a lower bound for the radius of $\partial\left(\Gamma_{\varepsilon}\right)$, we introduce the following notation:

$$
\begin{aligned}
s & =\left\|S_{0}\right\|, \quad p=\left\|V_{0} P_{0}\right\|, \quad q=\left\|V_{0} S_{0}\right\|, \\
r^{2} & =\sup \left\{\left\|V_{0} S_{0}^{k+1} V_{0} S_{0}\right\| /\left\|S_{0}\right\|^{k}, k=0,1,2, \ldots\right\} .
\end{aligned}
$$

LEMMA 2.1. Let $0<\varepsilon<1$. The radius of $\partial\left(\Gamma_{\varepsilon}\right)$ is at least

$$
\left[\frac{p^{2} s^{2}}{\varepsilon^{2}}+\frac{2 p s q}{\varepsilon(1-\varepsilon)}+\frac{r^{2}}{(1-\varepsilon)^{2}}\right]^{-1 / 2} \text {. }
$$

The convergence radii of the power series for $P(t)$ and $\hat{\lambda}(t)$ about 0 are at least equal to

$$
m_{0}=\max _{0<\varepsilon<1}\left[\frac{p^{2} s^{2}}{\varepsilon^{2}}+\frac{2 p s q}{\varepsilon(1-\varepsilon)}+\frac{r^{2}}{(1-\varepsilon)^{2}}\right]^{-1 / 2} .
$$

Proof. Since $\lambda_{0}$ is semisimple, we have $T_{0} P_{0}=\lambda_{0} P_{0}$. Hence $\lambda_{0}$ is a pole of order 1 of $R_{0}(z)$. The Laurent expansion of $R_{0}(z)$ about $\lambda_{0}$ is given by

$$
R_{0}(z)=\sum_{k=0}^{\infty} S_{0}^{k+1}\left(z-\lambda_{0}\right)^{k}-\frac{P_{0}}{z-\lambda_{0}}
$$


for $0<\left|z-\lambda_{0}\right|<\operatorname{dist}\left(\lambda_{0}, \sigma\left(T_{0}\right) \backslash\left\{\lambda_{0}\right\}\right)$ (compare [2, (5.18)]. Writing $R_{0}(z)=$ $R_{0}(z) P_{0}+R_{0}(z)\left(I-P_{0}\right)$, we see that

$$
\left[V_{0} R_{0}(z)\right]^{2}=A(z)+B_{1}(z)+B_{2}(z)+C(z),
$$

where

$$
\begin{aligned}
A(z) & =\left[V_{0} R_{0}(z) P_{0}\right]^{2}=\left(V_{0} P_{0}\right)^{2} /\left(z-\lambda_{0}\right)^{2} \\
B_{1}(z) & =V_{0} R_{0}(z) P_{0} V_{0} R_{0}(z)\left(I-P_{0}\right) \\
& =-\sum_{k=0}^{\infty} V_{0} P_{0} V_{0} S_{0}^{k+2}\left(z-\lambda_{0}\right)^{k}-\left(V_{0} P_{0} V_{0} S_{0}\right) /\left(z-\lambda_{0}\right) \\
B_{2}(z) & =V_{0} R_{0}(z)\left(I-P_{0}\right) V_{0} R_{0}(z) P_{0} \\
& =-\sum_{k=0}^{\infty} V_{0} S_{0}^{k+2} V_{0} P_{0}\left(z-\lambda_{0}\right)^{k}-\left(V_{0} S_{0} V_{0} P_{0}\right) /\left(z-\lambda_{0}\right)
\end{aligned}
$$

and

$$
C(z)=\left[V_{0} R_{0}(z)\left(I-P_{0}\right)\right]^{2}=\sum_{k=0}^{\infty} \sum_{j=0}^{\infty} V_{0} S_{0}^{k+1} V_{0} S_{0}^{j+1}\left(z-\lambda_{0}\right)^{k+j} .
$$

For $z \in \Gamma_{\varepsilon}$, we have $\left|z-\lambda_{0}\right|=\varepsilon / s$, so that

$$
\|A(z)\|=\frac{p^{2} s^{2}}{\varepsilon^{2}}, \quad\left\|B_{1}(z)\right\|,\left\|B_{2}(z)\right\| \leq p q s\left(\frac{1}{1-\varepsilon}+\frac{1}{\varepsilon}\right)=\frac{p s q}{\varepsilon(1-\varepsilon)},
$$

and

$$
\begin{aligned}
\|C(z)\| & \leq\left(\sum_{k=0}^{\infty}\left\|V_{0} S_{0}^{k+1} V_{0} S_{0}\right\|\left|z-\lambda_{0}\right|^{k}\right)\left(\sum_{j=0}^{\infty} s^{j}\left|z-\lambda_{0}\right|^{j}\right) \\
& \leq r^{2} /\left(1-\left|z-\lambda_{0}\right| s\right)^{2}=r^{2} /(1-\varepsilon)^{2},
\end{aligned}
$$

as $\left\|V_{0} S_{0}^{k+1} V_{0} S_{0}\right\| / s^{k} \leq r^{2}$ for $k=0,1,2, \ldots$. Thus, it follows that for $z \in \Gamma_{\varepsilon}$

$$
\left\|\left[V_{0} R_{0}(z)\right]^{2}\right\| \leq \frac{p^{2} s^{2}}{\varepsilon^{2}}+\frac{2 p s q}{\varepsilon(1-\varepsilon)}+\frac{r^{2}}{(1-\varepsilon)^{2}} .
$$

Also, by the spectral radius formula,

$$
r_{\sigma}\left(V_{0} R_{0}(z)\right)=\inf _{n=1,2, \ldots}\left\|\left[V_{0} R_{0}(z)\right]^{n}\right\|^{1 / n} \leq\left\|\left[V_{0} R_{0}(z)\right]^{2}\right\|^{1 / 2} .
$$

As the radius of $\partial\left(\Gamma_{\varepsilon}\right)$ is $\min _{z \in \Gamma_{\varepsilon}} 1 / r_{\sigma}\left(V_{0} R_{0}(z)\right)$, we see that it must at least be

$$
\left[\frac{p^{2} s^{2}}{\varepsilon^{2}}+\frac{2 p s q}{\varepsilon(1-\varepsilon)}+\frac{r^{2}}{(1-\varepsilon)^{2}}\right]^{-1 / 2} \text {. }
$$


Since for every $\varepsilon$ in $(0,1)$, the functions $P(t)$ and $\hat{\lambda}(t)$ are analytic on $\partial\left(\Gamma_{\varepsilon}\right)$, it follows that their power series converge if $|t|$ is less than $m_{0}$, given by (4).

REMARK 2.2. Letting $\varepsilon=\sqrt{p s} /(\sqrt{p s}+\sqrt{q})$, we see that

$$
(\sqrt{p s}+\sqrt{q})^{-1}\left[p s+2 \sqrt{p s q}+r^{2} / q\right]^{-1 / 2} \leq m_{0} .
$$

Since $r^{2} \leq\left\|V_{0} S_{0}\right\|^{2}=q^{2}$, it follows that (6) improves upon the lower bound $(\sqrt{p s}+\sqrt{q})^{-2}$ given by Kato [2, (3.21)]. This bound was obtained by majorizing the series

$$
V_{0} R_{0}(z)=\frac{-V_{0} P_{0}}{z-\lambda_{0}}+\sum_{k=0}^{\infty} V_{0} S_{0}^{k+1}\left(z-\lambda_{0}\right)^{k}
$$

term by term by the power series for the function

$$
\Phi_{1}\left(z-\lambda_{0}\right)=\frac{p}{z-\lambda_{0}}+\frac{q}{1-s\left(z-\lambda_{0}\right)} .
$$

(See [2, (3.14)].) On the other hand, the lower bound $m_{0}$ given by (4) is obtained by majorizing the series for $\left[V_{0} R_{0}(z)\right]^{2}$ by the power series for the function

$$
\Phi_{2}\left(z-\lambda_{0}\right)=\frac{p}{\left(z-\lambda_{0}\right)^{2}}+\frac{2 p q}{\left(z-\lambda_{0}\right)\left[1-s\left(z-\lambda_{0}\right)\right]}+\frac{r^{2}}{\left[1-s\left(z-\lambda_{0}\right)\right]^{2}} .
$$

THEOREM 2.3. The convergence radii of the power series for $P(t)$ and $\hat{\lambda}(t)$ are at least equal to $1 / 4 u$, where

$$
u=\max \{p s, \sqrt{p s q}, r\} .
$$

If $|t|<1 / 4 u$, then the operator $T(t)=T_{0}+t V_{0}$ has $m$ eigenvalues $\lambda_{1}(t), \ldots$, $\lambda_{m}(t)$, counted according to their algebraic multiplicities, and they satisfy

$$
\left|\lambda_{i}(t)-\lambda_{0}\right| \leq \frac{1-\sqrt{1-4 u|t|}}{2 s}, \quad 1 \leq i \leq m .
$$

Further, $\lambda_{1}(t), \ldots, \lambda_{m}(t)$ are the only spectral values of $T(t)$ lying in the open disk

$$
\left\{z \in \mathbb{C}:\left|z-\lambda_{0}\right|<\frac{1+\sqrt{1-4 u|t|}}{2 s}\right\} .
$$

Proof. Let $0<\varepsilon<1$. Then by Lemma 2.1, the radius of the disk $\partial\left(\Gamma_{\varepsilon}\right)$ is at least

$$
\left[\frac{u^{2}}{\varepsilon^{2}}+\frac{2 u^{2}}{\varepsilon(1-\varepsilon)}+\frac{u^{2}}{(1-\varepsilon)^{2}}\right]^{-1 / 2}=\frac{\varepsilon(1-\varepsilon)}{u} .
$$


It can be easily seen that $|t|<\varepsilon(1-\varepsilon) / u$ if and only if $u_{1}(t)<\varepsilon<u_{2}(t)$, where

$$
u_{1}(t)=\frac{1-\sqrt{1-4 u|t|}}{2} \text { and } u_{2}(t)=\frac{1+\sqrt{1-4 u|t|}}{2} .
$$

Hence for every $\varepsilon$ satisfying $u_{1}(t)<\varepsilon<u_{2}(t)$, we see that $t \in \partial\left(\Gamma_{\varepsilon}\right)$. Thus, $\Gamma_{\varepsilon}$ contains $m$ eigenvalues $\lambda_{1}(t), \ldots, \lambda_{m}(t)$ of $T(t)$ (counted according to their algebraic multiplicities) and does not contain any other spectral value of $T(t)$. In particular,

$$
\left|\lambda_{i}(t)-\lambda_{0}\right|<\varepsilon / s, \quad i=1, \ldots, m .
$$

Letting $\varepsilon \rightarrow u_{1}(t)$, we have

$$
\left|\lambda_{i}(t)-\lambda_{0}\right| \leq \frac{1-\sqrt{1-4 u|t|}}{2 s}, \quad i=1, \ldots, m,
$$

while letting $\varepsilon \rightarrow u_{2}(t)$, we find that the open disk

$$
\left\{z \in \mathbb{C}:\left|z-\lambda_{0}\right|<\frac{1+\sqrt{1-4 u|t|}}{2 s}\right\}
$$

does not contain any other spectral value of $T(t)$.

REMARK 2.4. The functions $u_{1}(t)$ and $u_{2}(t)$ introduced in (10) satisfy $0 \leq u_{1}(t) \leq \frac{1}{2} \leq u_{2}(t) \leq 1$ for $|t|<1 / 4 u$, and they depend only on $|t|$. As $|t| \downarrow 0, u_{1}(t)$ monotonically decreases to 0 , while $u_{2}(t)$ monotonically increases to 1 , and as $|t| \uparrow 1 / 4 u, u_{1}(t)$ monotonically increases to $\frac{1}{2}$, while $u_{2}(t)$ monotonically decreases to $\frac{1}{2}$. Thus, a smaller absolute value of the parameter $t$ (corresponding to a smaller perturbation $t V_{0}$ ) yields a better estimate for $\left|\lambda_{i}(t)-\lambda_{0}\right|, i=1, \ldots, m$, and at the same time a larger disk of isolation of $\left\{\lambda_{1}(t), \ldots, \lambda_{m}(t)\right\}$ from the rest of $\sigma(T(t))$. For all $t$ with $|t|<1 / 4 u$, we have

$$
\left\{z \in \mathbb{C}:\left|z-\lambda_{0}\right|<\frac{1}{2 s}\right\} \cap \sigma(T(t))=\left\{\lambda_{1}(t), \ldots, \lambda_{m}(t)\right\} .
$$

These phenomena are illustrated in Figure 1.

REMARK 2.5. The lower bounds

$$
(\sqrt{p s}+\sqrt{q})^{-1}\left[p s+2 \sqrt{p s q}+\frac{r^{2}}{q}\right]^{-1 / 2} \text { and } \frac{1}{4 \max \{p s, \sqrt{p s q}, r\}}
$$

for the convergence radii of the series for $P(t)$ and $\hat{\lambda}(t)$ obtained in Remark 2.2 and Theorem 2.3 are significant in the operator approximation theory. We note that both these bounds tend to infinity if $p$ and $r$ approach 0 , 

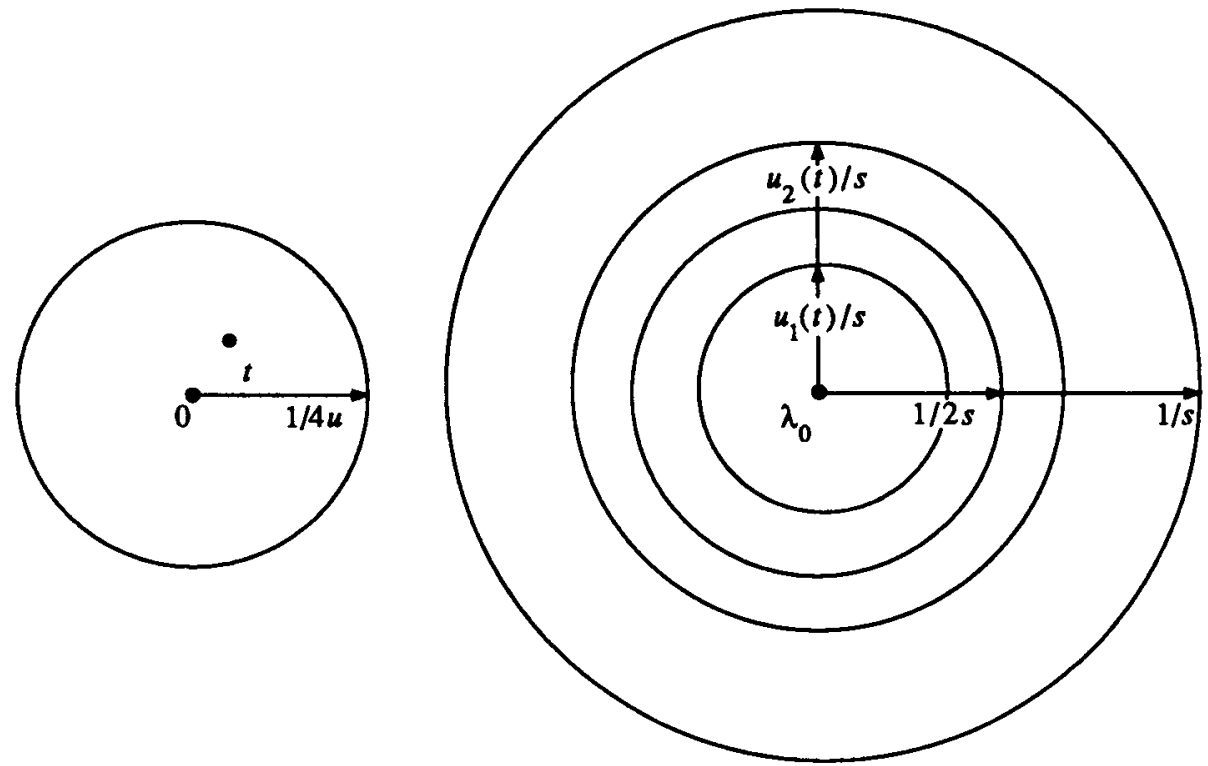

Figure 1

and if $s$ and $q$ remain bounded. Let a compact operator $T$ on $X$ be approximated by a sequence of bounded operators $\left(T_{n}\right)$ in a collectively compact manner (that is, $T_{n} x \rightarrow T x$ for every $x \in X$ and the set $\bigcup_{n=1}^{\infty}\{(T-$ $\left.\left.T_{n}\right) x: x \in X,\|x\| \leq 1\right\}$ is totally bounded). Let $\lambda$ be a simple eigenvalue of $T$ such that $|\lambda|>\operatorname{dist}(\lambda, \sigma(T) \backslash\{\lambda\})$. It is shown in [3, Theorem 2.1] and [5, Theorem 3.4] that for all large $n, T_{n}$ has a simple eigenvalue $\lambda_{n}$ such that $\lambda_{n} \rightarrow \lambda$, and if we let $T_{0}=T_{n}, V_{0}=T-T_{n}, \lambda_{0}=\lambda_{n}, s_{n}=\left\|S_{n}\right\|, p_{n}=$ $\left\|V_{n} P_{n}\right\|, q_{n}=\left\|V_{n} S_{n}\right\|$ and

$$
r_{n}^{2}=\sup \left\{\left\|V_{n} S_{n}^{k+1} V_{n} S_{n}\right\| /\left\|S_{n}\right\|^{k}: k=0,1,2, \ldots\right\},
$$

then $p_{n} \rightarrow 0$ and $r_{n} \rightarrow 0$, while $\left(s_{n}\right)$ and $\left(q_{n}\right)$ remain bounded as $n \rightarrow \infty$. The above lower bounds then guarantee that for a suitably large fixed $n$, the point 1 lies in the convergence disks of $P(t)$ and $\hat{\lambda}(t)$, and hence the spectral projection $P(1)$ and the eigenvalue $\lambda=\lambda(1)$ of $T(1)=T$ can be approximated by the partial sums of the power series for $P(t)$ and $\hat{\lambda}(t)$, respectively.

We note that since $\left(q_{n}\right)$ may not tend to zero as $n \rightarrow \infty$, the lower bound $\left(\sqrt{p_{n} s_{n}}+\sqrt{q_{n}}\right)^{-2}$ for the convergence radii of $P(t)$ and for $\lambda(t)$ obtained by Kato is not useful for ascertaining the convergence at $t=1$. In case $\left(T_{n}\right)$ converges to $T$ in the norm, then $\left(q_{n}\right)$ does tend to zero as $n \rightarrow \infty$, and Kato's lower bound becomes applicable. We mention that there are many important cases where $\left(T_{n}\right)$ approximates $T$ in the collectively compact 
manner, but not in the norm. For example, for a nonzero compact Fredholm integral operator $T$ on $X=C([a, b])$, let $\left(T_{n}\right)$ be a sequence of Nyström or Fredholm approximations of $T$, or if $\pi_{n}$ is an interpolatory projection on $X$ with $\pi_{n} x \rightarrow x$ for all $x \in X$, let $T_{n}=T \pi_{n}$ or $\pi_{n} T \pi_{n}$ ([1, pages 18-19] and [4, pages 292-294]).

In case $T_{0}$ is a normal operator on a Hilbert space $X$, then every eigenvalue of $T_{0}$ of finite algebraic multiplicity is semisimple. For this case, we refer to [4, Theorem 10.6] and [2, Theorem 3.9] for an especially elegant analogue of Theorem 2.3.

\section{Convergence radius of an eigenvector series}

Let $\lambda_{0}$ be a simple eigenvalue of $T_{0}$, that is, $\lambda_{0}$ is a semisimple eigenvalue and the associated eigenspace is one dimensional. Then for $t \in \partial\left(\Gamma_{0}\right), P(t)$ is of rank 1, and the operator $T(t)=T_{0}+t V_{0}$ has only one simple eigenvalue $\lambda(t)$ lying inside $\Gamma_{0}$, so $\hat{\lambda}(t)=\lambda(t)$ is analytic for $t \in \partial\left(\Gamma_{0}\right)$. Let $\phi_{0}$ be an eigenvector of $T_{0}$ corresponding to $\lambda_{0}$. Then the adjoint operator $T_{0}^{*}$ is closed and densely defined in the adjoint space $X^{*}=\left\{x^{*}: X \rightarrow\right.$ $\mathbb{C} ; x^{*}$ is conjugate linear and continuous $\}$. There is a unique eigenvector $\phi_{0}^{*}$ of $T_{0}^{*}$ corresponding to $\bar{\lambda}_{0}$ such that $\left\langle\phi_{0}, \phi_{0}^{*}\right\rangle=1$ and we have

$$
P_{0} x=\left\langle x, \phi_{0}^{*}\right\rangle \phi_{0}, \quad x \in X .
$$

As mentioned in the introduction, the function

$$
\phi(t)=P(t) \phi_{0} /\left\langle P(t) \phi_{0}, \phi_{0}^{*}\right\rangle
$$

is meromorphic in $\partial\left(\Gamma_{0}\right)$ and $\phi(0)=\phi_{0}$. If $t_{0} \in \partial\left(\Gamma_{0}\right)$ is not a pole of the function $\phi$, then $\phi\left(t_{0}\right)$ is an eigenvector of $T\left(t_{0}\right)$ corresponding to $\lambda\left(t_{0}\right)$. We have not been able to decide whether the function $\phi$ ever has a pole in $\partial\left(\Gamma_{0}\right)$. Nevertheless, we shall obtain a subregion of $\partial\left(\Gamma_{0}\right)$ which contains 0 and which is free of any pole of the function $\phi$.

Lemмa 3.1. Let $t_{0} \in \partial\left(\Gamma_{0}\right)$. Then there is a unique nonnegative integer $k_{0}$ such that

$$
\psi\left(t_{0}\right)=\lim _{t \rightarrow t_{0}} P(t) \phi_{0} /\left(t-t_{0}\right)^{k_{0}}
$$

is an eigenvector of $T\left(t_{0}\right)$ corresponding to $\lambda\left(t_{0}\right)$. Further, $t_{0}$ is a pole of the function $\phi$ if and only if $\left\langle\psi\left(t_{0}\right), \phi_{0}^{*}\right\rangle=0$, and in this case, $\psi\left(t_{0}\right)$ is an eigenvector also of the operator $\left(I-P_{0}\right) T\left(t_{0}\right)_{\mid\left(I-P_{0}\right) D_{0}}$. 
Proof. Let $t_{0} \in \partial\left(\Gamma_{0}\right)$. Since $P(0) \phi_{0}=\phi_{0} \neq 0$, the $X$-valued analytic function $t \mapsto P(t) \phi_{0}$ is not identically zero on $\partial\left(\Gamma_{0}\right)$. Hence either it has an isolated zero of finite order at $t_{0}$ or it is nonzero at $t_{0}$. In any case, there is a unique nonnegative integer $k_{0}$ and an analytic function $\psi$ in a neighbourhood $U$ of $t_{0}$ such that $P(t) \phi_{0}=\left(t-t_{0}\right)^{k_{0}} \psi(t)$ and $\psi\left(t_{0}\right) \neq 0$. For $t \in U$, we have

$$
\left\langle P(t) \phi_{0}, \phi_{0}^{*}\right\rangle=\left(t-t_{0}\right)^{k_{0}}\left\langle\psi(t), \phi_{0}^{*}\right\rangle .
$$

Hence the function

$$
\phi(t)=\frac{\psi(t)}{\left\langle\psi(t), \phi_{0}^{*}\right\rangle}, \quad t \neq t_{0},
$$

has a pole at $t_{0}$ if and only if $\left\langle\psi\left(t_{0}\right), \phi_{0}^{*}\right\rangle=0$. Clearly, $\lim _{t \rightarrow t_{0}} \psi(t)$ exists and equals $\psi\left(t_{0}\right)$. Now,

$$
\psi(t)=P(t) \phi_{0} /\left(t-t_{0}\right)^{k_{0}}, \quad t \in U, t \neq t_{0},
$$

is an eigenvector of $T(t)$ corresponding to $\lambda(t)$. The continuity of the functions $t \mapsto P(t)$ and $t \mapsto \psi(t)$ at $t=t_{0}$ shows that $\psi\left(t_{0}\right)$ is an eigenvector of $T\left(t_{0}\right)$ corresponding to $\lambda\left(t_{0}\right)$.

Assume now that $t_{0}$ is a pole of the function $\phi$. Then

$$
P_{0} \psi\left(t_{0}\right)=\left\langle\psi\left(t_{0}\right), \phi_{0}^{*}\right\rangle \phi_{0}=0,
$$

that is, $\psi\left(t_{0}\right) \in\left(I-P_{0}\right) D_{0}$. Since

$$
\left(I-P_{0}\right) T\left(t_{0}\right) \psi\left(t_{0}\right)=\lambda\left(t_{0}\right)\left(I-P_{0}\right) \psi\left(t_{0}\right)=\lambda\left(t_{0}\right) \psi\left(t_{0}\right),
$$

it is apparent that $\psi\left(t_{0}\right)$ is an eigenvector of $\left(I-P_{0}\right) T\left(t_{0}\right)_{\mid\left(I-P_{0}\right) D_{0}}$ corresponding to $\lambda\left(t_{0}\right)$.

Proposition 3.2. The meromorphic function $\phi$ given by (11) has no pole in the open set

$$
G=\left\{t \in \partial\left(\Gamma_{0}\right): s\left|\lambda(t)-\lambda_{0}\right|+r|t|<1\right\} .
$$

For every $t \in G, \phi(t)$ is an eigenvector of $T(t)$ corresponding to $\lambda(t)$.

Proof. Let $t \in G$. We show that $\lambda(t)$ is not in the spectrum of the operator $\left(I-P_{0}\right)\left(T_{0}+t V_{0}\right)_{\mid\left(I-P_{0}\right) D_{0}}=\left(I-P_{0}\right) T(t)_{\mid\left(I-P_{0}\right) D_{0}}$. Lemma 3.1 then implies the desired result.

Let us denote $\left(I-P_{0}\right) D_{0}$ by $Z$ and $\lambda(t)$ by $z$ for brevity. Since $t \in G$, we have $\left|z-\lambda_{0}\right|<1 / \mathrm{s}$. By (2), $\lambda_{0}$ is the only spectral value of $T_{0}$ in the open disk with centre $\lambda_{0}$ and radius $1 / s$, and $\sigma\left(T_{0 \mid Z}\right)=\sigma\left(T_{0}\right) \backslash\left\{\lambda_{0}\right\}$. Hence 
it follows that $z \notin \sigma\left(T_{0 \mid Z}\right)$. In order to conclude $z \notin \sigma\left(I-P_{0}\right)\left(T_{0}+t V_{0}\right)_{\mid Z}$, it is enough to show that $r_{\sigma}(E(z))<1$, where

$$
E(z)=\left[T_{0 \mid Z}-\left(I-P_{0}\right)\left(T_{0}+t V_{0}\right)_{\mid Z}\right]\left[T_{0 \mid Z}-z I_{\mid Z}\right]^{-1} \text {. }
$$

Now,

$$
E\left(\lambda_{0}\right)=-t\left(I-P_{0}\right) V_{0} S_{0 \mid Z}
$$

and if $z \neq \lambda_{0}$, then

$$
E(z)=-t\left(I-P_{0}\right) V_{0} R_{0}(z)_{\mid z}
$$

In the first case,

$$
\begin{aligned}
r_{\sigma}\left(E\left(\lambda_{0}\right)\right) & \leq|t| r_{\sigma}\left(\left(I-P_{0}\right) V_{0} S_{0}\right)=|t| r_{\sigma}\left(V_{0} S_{0}\left(I-P_{0}\right)\right) \\
& =|t| r_{\sigma}\left(V_{0} S_{0}\right) \leq|t|\left\|\left(V_{0} S_{0}\right)^{2}\right\|^{1 / 2} \leq|t| r,
\end{aligned}
$$

which is less than 1 since $t \in G$. In the second case, that is, when $z \neq \lambda_{0}$,

$$
\begin{aligned}
r_{\sigma}(E(z)) & \leq|t| r_{\sigma}\left(\left(I-P_{0}\right) V_{0} R_{0}(z)\right)=|t| r_{\sigma}\left(V_{0} R_{0}(z)\left(I-P_{0}\right)\right) \\
& =|t|\|C(z)\|^{1 / 2} \leq|t| r /\left(1-s\left|z-\lambda_{0}\right|\right),
\end{aligned}
$$

by (5). Since $z=\lambda(t)$ and $t \in G$, it again follows that $r_{\sigma}(E(z))<1$.

Theorem 3.3. Let $0<\varepsilon<1$ and $t \in \mathbb{C}$ be such that

$$
|t|<\left[\frac{p^{2} s^{2}}{\varepsilon^{2}}+\frac{2 p s q}{\varepsilon(1-\varepsilon)}+\frac{r^{2}}{(1-\varepsilon)^{2}}\right]^{-1 / 2} .
$$

Then $\phi(t)=P(t) \phi_{0} /\left\langle P(t) \phi_{0}, \phi_{0}^{*}\right\rangle$ is an eigenvector of $T(t)$ corresponding to $\lambda(t)$ such that $\left\langle\phi(t), \phi_{0}^{*}\right\rangle=1$. The radius of convergence of the power series for $\phi(t)$ about 0 is at least equal to $m_{0}$ given by (4).

Proof. By Lemma 2.1, $t$ belongs to $\partial\left(\Gamma_{\varepsilon}\right)$, so that $\left|\lambda(t)-\lambda_{0}\right|<\varepsilon / s$. Hence

$$
s\left|\lambda(t)-\lambda_{0}\right|+r|t|<\varepsilon+\frac{r}{\left[\frac{p^{2} s^{2}}{\varepsilon^{2}}+\frac{2 p s q}{\varepsilon(1-\varepsilon)}+\frac{r^{2}}{(1-\varepsilon)^{2}}\right]^{1 / 2}}<1 .
$$

By Proposition 3.2, we see that $\phi(t)$ is an eigenvector of $T(t)$ corresponding to $\lambda(t)$. It is obvious that $\left\langle\phi(t), \phi_{0}^{*}\right\rangle=1$. Thus, the function $\phi$ is analytic on

$$
\left\{t \in \mathbb{C}:|t|<\left[\frac{p^{2} s^{2}}{\varepsilon^{2}}+\frac{2 p s q}{\varepsilon(1-\varepsilon)}+\frac{r^{2}}{(1-\varepsilon)^{2}}\right]^{-1 / 2}\right\}
$$

for every $\varepsilon, 0<\varepsilon<1$. The desired result now follows from the very definition of $m_{0}$. 
Remark 3.4. As the quantities

$$
(\sqrt{p s}+\sqrt{q})^{-1}\left[p s+2 \sqrt{p s q}+\frac{r^{2}}{q}\right]^{-1 / 2} \text { and } \frac{1}{4 \max \{p s, \sqrt{p s q}, r\}}
$$

are at most equal to $m_{0}$, they also give lower bounds for the convergence radius of the power series for $\phi(t)$, just as we had noted this for $P(t)$ and $\lambda(t)$. The lower bound $(\sqrt{p s}+\sqrt{q})^{-2}$ for $\phi(t)$ given in [2, Problem 3.7] (obtained by finding a majorizing series for $\phi(t)$ ) is thus improved to $(\sqrt{p s}+\sqrt{q})^{-1}\left[p s+2 \sqrt{p s q}+r^{2} / q\right]^{1 / 2}$. (Note $r \leq q$.)

The lower bound $1 /(4 \max \{p s, \sqrt{p s q}, r\})$ for the convergence radii of the series for $\lambda(t)$ and for $\phi(t)$ is obtained in [5] by considering estimates for the iteratively defined $n$th terms of the power series for $\lambda(t)$ and $\phi(t)$, while in [7, pages 82 and 85], a similar lower bound is obtained by considering the power series for $P(t)$.

\section{References}

[1] P. M. Anselone, Collectively compact operator approximation theory, (Prentice-Hall, Englewood Cliffs, N. J., 1971).

[2] T. Kato, Perturbation theory for linear operators, 2nd ed. (Springer-Verlag, Berlin, Heidelberg, New York and Tokyo, 1976).

[3] R. P. Kulkarni and B. V. Limaye, 'On the error estimates for the Rayleigh-Schrödinger series and the Kato-Rellich perturbation series', J. Austral. Math. Soc. Ser. A 46 (1989), 456-468.

[4] B. V. Limaye, Spectral perturbation and approximation with numerical experiments, Proc. Centre Math. Anal., Vol. 13, (Australian National Univ., 1986).

[5] B. V. Limaye and M. T. Nair, 'On the accuracy of Rayleigh-Schrödinger approximation,' J. Math. Anal. Appl. 139 (1989), 413-431.

[6] M. T. Nair, 'A note on the Rayleigh-Schrödinger series', J. Math. Phys. Sci. 23 (1989), 185-193.

[7] P. Redont, Application de la théorie de la perturbation des opérateurs linéaires à l'obtention de bornes d'erreur sur les éléments propres et à leur calcul, (Thèse Doct.Ing., Univ. de Grenoble, 1979).

Indian Institute of Technology

Powai, Bombay 400076

University of Goa

India Santa Cruz, Goa 403005

India 